\title{
SGEM Hot Off the Press: hypertonic saline in severe traumatic brain injury: a systematic review and meta-analysis of randomized controlled trials
}

\author{
Eve Purdy, MD*; Brent Thoma, MD, MA ${ }^{\dagger}$; Ken Milne, MD, MSc ${ }^{\ddagger}$; Chris Bond, $\mathrm{MD}^{\S}$
}

\section{ABSTRACT}

As part of the Canadian Fournal of Emergency Medicine's (CFEM) developing social media strategy, ${ }^{1}$ we are collaborating with the Skeptics' Guide to Emergency Medicine (SGEM) to summarize and critically appraise the current emergency medicine (EM) literature using evidence-based medicine principles. In the "Hot Off the Press" series, we select original research manuscripts published in CFEM to be featured on the SGEM website/podcast and discussed by the study authors and the online EM community. A similar collaboration is under way between the SGEM and Academic Emergency Medicine. What follows is a summary of the selected article, the immediate post-publication synthesis from the SGEM podcast, commentary by the first author, and the subsequent discussion from the SGEM blog and other social media. Through this series, we hope to enhance the value, accessibility, and application of important, clinically relevant EM research. In this, the second SGEM HOP hosted collaboratively with CFEM, we discuss a systematic review evaluating the use of hypertonic saline in the treatment of severe traumatic brain injury. ${ }^{2}$

Keywords: traumatic brain injury, social media, twitter, mannitol

\section{INTRODUCTION}

Severe traumatic brain injuries (TBIs) are common in Canadian emergency departments. ${ }^{2}$ These injuries are associated with high morbidity and mortality. ${ }^{3}$ In addition to the damage from primary trauma, secondary cerebral injuries can occur as a result of increased intracranial pressure (ICP). Because raising ICP is a mechanism for mortality and poor neurologic outcomes, a number of interventions target this disease mechanism. ${ }^{4}$ Treatment approaches include, but are not limited to, cerebrospinal fluid drainage, decompressive craniectomy, and barbiturate coma. ${ }^{5}$ However, the firstline treatment most often consists of intravenous infusion of a hyperosmolar solution. ${ }^{6}$

Mannitol is the hyperosmolar solution recommended by multiple clinical practice guidelines and is considered to be the gold standard for hyperosmolar therapy. ${ }^{6}$ However, a well-known adverse effect of this treatment is diuresis, which can lead to hypotension, a state that is associated with higher mortality in TBI patients. ${ }^{7-9}$ Hypertonic saline solutions have been proposed as an alternative to mannitol because they have both volume repletion properties and provide the desired osmotic effect. Six previous systematic reviews have compared mannitol to hypertonic saline, but their validity has been questioned because they used low-sensitivity search strategies, did not include the most recent evidence, included non-randomized controlled trials (non-RCTs), and included patients with nontraumatic causes of elevated ICP. ${ }^{10-15}$

\section{SUMMARY}

Berger-Pelletier et al. conducted a literature search to identify RCTs comparing hypertonic saline to

From the *Department of Emergency Medicine, Queen's University, Kingston, ON; tDepartment of Emergency Medicine, University of Saskatchewan, Saskatoon, SK; ¥Division of Emergency Medicine, Western University, London, ON; and the §Department of Emergency Medicine, University of Calgary, Calgary, AB.

Correspondence to: Eve Purdy, Department of Emergency Medicine, Kingston General Hospital, c/o 76 Stuart Street, Kingston, ON K7L 2V7; Email: epurdy@qmed.ca 
hyperosmolar solutions (mannitol or sodium bicarbonate), iso-osmolar solutions (normal saline or ringer's lactate), or hypo-osmolar solutions. They searched MEDLINE, EMBASE, the Cochrane Central Register of Controlled Trials, Scopus, Web of Science, BIOSIS databases, grey literature, and the reference sections of selected articles in a search that was not limited by language.

They found 21 relevant studies, each of which was reviewed by two authors who independently extracted data. Eleven of these articles were included in the qualitative analysis, and nine were included in the quantitative analysis. The 11 studies that met the inclusion criteria on full-text review included 1820 patients (the vast majority of which were $>18$ years old) with TBIs. The risk of bias was performed using a standardized tool.

\section{QUALITY ASSESSMENT}

We used the Best Evidence in Emergency Medicine (BEEM) tool as a reference in our critical appraisal of this systematic review. ${ }^{16,17}$

This study addressed a clinically relevant, patientoriented outcome (mortality) as the primary outcome and a disease-oriented outcome (measured ICP) as a secondary outcome. The authors' search strategy was detailed and exhaustive. In particular, their exploration of the grey literature and inclusion of studies in all languages was particularly impressive. Two independent authors screened the titles and abstracts for a full-text review.

Unfortunately, most of the available RCTs were small in size. Of the 11 included studies, only 3 registered more than 100 patients, and 1 large study comprising 1282 patients made up $70 \%$ of the patient population. Only two of the studies included were at low risk of bias with the rest suffering from methodological flaws related to blinding and a lack of outcome data.

The authors planned a priori to evaluate secondary outcomes, including neurologic outcomes at the intensive care unit or hospital discharge and hospital length of stay. Unfortunately, a quantitative evaluation of these outcomes was not possible due to under-reporting.

\section{RESULTS}

The evidence for hypertonic saline for ICP in TBI, as reviewed by Berger-Pelletier et al., comes from a group of studies, most of which are quite small and at risk of bias. The authors conclude that hypertonic saline cannot be recommended as a first-line agent for managing patients with TBI.

\section{TAKE-TO-WORK POINTS}

Hypertonic saline does not confer mortality benefit or improvement on ICP control when compared to other solutions. Further research is needed to assess its non-inferiority to mannitol and to evaluate other considerations such as cost and effect on neurologic outcomes.

\section{METHODOLOGY}

The social media discussion started with the launch of the blog post and podcast ${ }^{18}$ on March 27, 2016, and continued for 1 week through April 3, 2016. An invitation to comment on the article was included in the audio of the podcast, the text of the blog post, and on social media communications (Twitter and Facebook). Throughout the week, multiple reposts of the article were made on Twitter. Social media responses written in the SGEM blog's comment section, the SGEM Facebook page, and on Twitter (directed at @thesgem, @socmobem, @ElysePelletier and/or, using the \#SGEMHOP hashtag) were reviewed by the authorship team.

Multiple metrics of dissemination were tracked by the SGEM HOP team:

- Blog post page views were monitored using the Jetpack plugin by Wordpress.com. ${ }^{19}$

- Facebook "reach" analytics were provided by Facebook and correlate to the number of users who saw the original SGEM Facebook page on their own newsfeed. Facebook likes were also tracked. ${ }^{20}$

- Twitter impressions (the number of users whose newsfeeds contained a tweet featuring the \#SGEMHOP hashtag) were tracked using Symplur, a software program that tracks health care related Twitter conversations. ${ }^{21}$ Tweets not containing the hashtag were not tracked by Symplur. The number of impressions is calculated by taking the number of tweets per Twitter user using the \#SGEMHOP hashtag and multiplying it by the number of followers that participant has.

- The altmetric score is a relatively new way to track the disseminative impact of research articles online by tracking mentions on social media 
(e.g., Facebook, Twitter) and mentions on blogs, podcasts, and news outlets. ${ }^{22}$ Limitations of the altmetric score include the inability to distinguish quality from popularity, "gaming" of dissemination through automated downloads and social media bots, and they do not capture those reading articles through traditional print media. ${ }^{23}$ The altmetric score of Berger-Pelletier's article was compared to articles from the same issue of CFEM, all other articles published in $C 7 E M$, research articles from all other outlets, and the article covered in the first CFEM-SGEM HOP. ${ }^{24,25}$

A thematic analysis, performed through an inductive approach, of blog comments and Twitter comments was carried out by E.P. Tweets and posts simply promoting the \#SGEMHOP article were not included in the thematic analysis. E.P. identified themes that captured key discussion points across platforms. Then all authors, through a consensus-based content analysis, decided on quotes from the blog posts that were representative of the discussion. Of note, all original tweets, comments, and content are available online for independent review.

\section{RESULTS OF THE SOCIAL MEDIA RESPONSE}

Table 1 presents the social media uptake of the second SGEM HOP. During the 1-week period, \#SGEMHOP was used in 103 tweets by 37 separate users. Thirty-three of these tweets were from this study's authors or the CFEM account prompting Twitter users to engage in conversation, linking to the original CFEM article and the \#SGEMHOP blog post. There was a bimodal distribution in the peak timing of Twitter activity correlating to the day of release and 3 days after release. Conversation using the hashtag effectively ended within 4 days. Although outside of the scope of our a priori 2-week analysis window, it should be noted that the online discussion was re-invigorated more than a month after the initial posting, resulting in six additional comments on the blog. The altmetric score for the article reached 46 . While lower than the last article featured for SGEMHOP (76), this is the fifth highest score for articles published in CFEM article and within the top 5 th percentile of altmetric scores for all published research (Figure 1). The mean altmetric score for other original research articles in this CFEM issue was 7 (1-46).

\section{ONLINE DISCUSSION SUMMARY}

There were many insightful comments on social media networks, with the best appearing directly on the SGEM blog. One Twitter user, @KirstyChallen, even made an infographic on the topic. Discussions across platforms had similar tones and content. Of particular note, study author Elyse Pelletier was engaged in all of the discussions on the blog, offering clarification

\begin{tabular}{|c|c|c|c|}
\hline $\begin{array}{l}\text { Social media } \\
\text { analytic }\end{array}$ & Metric & Metric definition & Count \\
\hline \multirow[t]{2}{*}{$\begin{array}{l}\text { The SGEM blog } \\
\text { statistics }\end{array}$} & Number of page views & $\begin{array}{l}\text { Number of times the webpage containing the post was } \\
\text { viewed }\end{array}$ & 1609 \\
\hline & $\begin{array}{l}\text { Number of blog } \\
\text { comments }\end{array}$ & $\begin{array}{l}\text { Comments made directly on the website in the blog } \\
\text { comments section }\end{array}$ & $\begin{array}{l}10 \text { (average } 80 \text { words/post) - } 7 \text { of which } \\
\text { were new comments, not replies by the } \\
\text { original study author }\end{array}$ \\
\hline \multirow[t]{4}{*}{ Symplur analytics } & Number of tweets & Number of tweets containing the hashtag \#SGEMHOP & 103 \\
\hline & $\begin{array}{l}\text { Number of Twitter } \\
\text { participants }\end{array}$ & $\begin{array}{l}\text { Number of unique Twitter users who participated with } \\
\text { tweeting during } 1 \text { week around the event }\end{array}$ & 37 \\
\hline & Twitter impressions & $\begin{array}{l}\text { The number of potential views of a tweet or a tweet } \\
\text { containing a specific hashtag in users' Twitter streams, } \\
\text { as calculated by the number of tweets per participant } \\
\text { and multiplying it with the number of followers that } \\
\text { participant has. }\end{array}$ & The \#SGEMHOP hashtag: 207, 728 \\
\hline & & & Twitter poll: 997 \\
\hline \multirow[t]{2}{*}{ Facebook statistics } & Reach & $\begin{array}{l}\text { The number of users whose newsfeed featured the } \\
\text { original SGEM post }\end{array}$ & 1749 \\
\hline & Likes & $\begin{array}{l}\text { The number of users who "liked" the official SGEM post } \\
\text { pages (does not include likes of other posts sharing the } \\
\text { article) }\end{array}$ & 7 \\
\hline
\end{tabular}




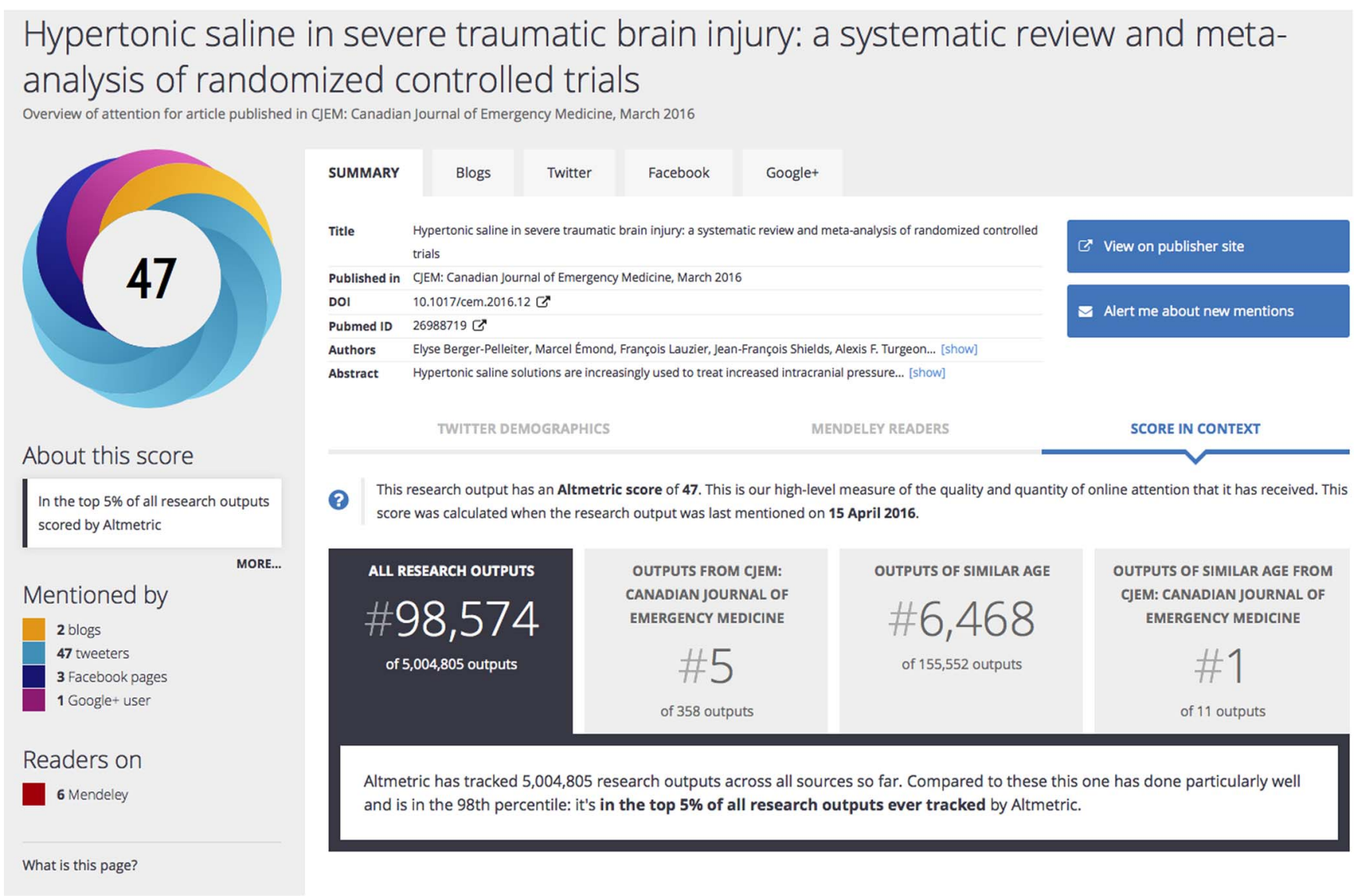

Figure 1. Screen capture of the altmetrics data. Available at: https://cambridgejournals.altmetric.com/details/6255240\#score (accessed 16 April 2016).

regarding her study and clinical expertise regarding the use of hypertonic fluid therapy in TBI.

Overall, it was felt that the level of evidence supporting the use of hypertonic fluids in TBIs is inadequate. Justin Morgenstern highlighted the fact that, although this systematic review tempers some of the recent excitement around hypertonic saline, we should keep in mind that mannitol became the gold standard through a much less rigorous process of evidence acceptance. Brent Thoma questioned whether we have enough evidence to recommend mannitol as first-line therapy, linking to a Cochrane review showing a non-significant relative risk of 0.83 (95\% CI 0.47 to 1.46 ) for mortality. ${ }^{7}$ Nadim Lalani offered a sobering reminder that the rate of mortality in this population is very high and suggested that future studies should include outcomes related to neurologic outcome in patients who do survive. These respondents agreed that the outcomes of future trials treatment should have patient-oriented (not diseaseoriented) outcomes and also include a more in-depth evaluation of adverse events, because these would likely be the impetus for a change in practice.

Questions were also raised surrounding the practicalities and costs associated with delivering these treatments. For example, Ryan Deedo, a flight physician, provided a practical perspective, noting that hypertonic saline is easier to store than mannitol, potentially making it a better choice in prehospital environments. This served as a reminder that evidence has to be interpreted within the context of which we work.

Interestingly, the Twitter poll, Figure 2, with 53 participants found that many physicians use hypertonic saline, a practice that differs from the authors' conclusion that hypertonic saline should not be recommended as first-line therapy. Because the Twitter poll sampled a small number of unique emergency physicians, it may not be representative of the broader EM community; however, it suggests that at least some emergency physicians disagree with the authors' conclusions and support the further study of this question. 
Following

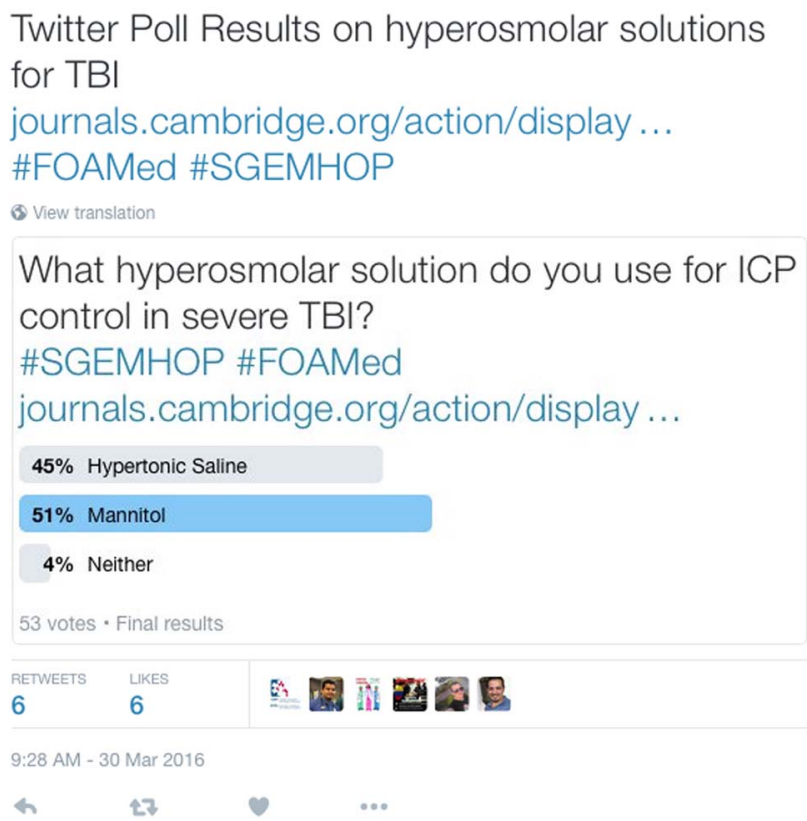

Figure 2. Tweet from Ken Milne (@theSGEM) containing a poll on what hypertonic solution respondents use for traumatic brain injury.

There are several limitations to this study. First, we recognize that there is sampling bias of physicians engaging in social media discussions. We appreciate that those online likely represent a unique subset of emergency physicians who cannot be extrapolated to be representative of the entire profession. Furthermore, there were 37 unique contributions to the online Twitter discussion, which represents less than $5 \%$ of emergency physicians with a Twitter account. ${ }^{26}$ Second, Twitter engagement captured only those discussions that include the \#SGEMHOP hashtag, and it is possible that we missed other important discussions on the topic online. There were a relatively small number of comments on the blog given the large number of times it was viewed. In order to increase engagement, we could consider posing questions to readers with a time-delayed release of the \#SGEMHOP expert commentary. Alternatively, we could re-evaluate the release date/time to maximize the likelihood that individuals will respond. The Jetpack software used to collect website analytics did not allow for the collection of some useful metrics that could better help characterize engagement (e.g., time spent on page and the geographic location of participants). It would also be useful to track the number of article downloads from the CFEM website. In future study of online journal club models, we would suggest using analytical software that is capable of capturing these metrics.

\section{CONCLUSION}

The second collaborative knowledge translation effort between CFEM and the SGEM was successful on a number of fronts. First, the featured article had higher altmetric scores than other original research articles in the same CFEM issue, lending support to the hypothesis that a targeted social media strategy promotes dissemination. Second, direct access to the author of this study through the comments section on the SGEM allowed readers the unique opportunity to seek clarification on the paper and the overall topic, even months after the initial discussion was posted. Third, the Twitter poll highlighted possible discordance between the authors' conclusions that hypertonic saline cannot be recommended as first-line therapy and some physicians' clinical utilization. Finally, the discussion on the blog led to the suggestion of several future directions of research on this topic.

Competing interests: None declared.

\section{REFERENCES}

1. Thoma B, Mohindra R, Artz JD, et al. CFEM and the changing landscape of medical education and knowledge translation. CFEM 2015;17(2):184-7.

2. Zygun DA, Laupland KB, Hader WJ, et al. Severe traumatic brain injury in a large Canadian health region. Can 7 Neurol Sci 2005;32(1):87-92.

3. Turgeon AF, Lauzier F, Simard J-F, et al. Mortality associated with withdrawal of life-sustaining therapy for patients with severe traumatic brain injury: a Canadian multicentre cohort study. CMA7 2011;183(14):1581-8.

4. Park E, Bell JD, Baker AJ. Traumatic brain injury: can the consequences be stopped? CMA7 2008;178(9):1163-70.

5. Bullock R, Chestnut R, Ghajar J, et al. Guidelines for the surgical management of traumatic brain injury. Neurosurgery 2006;58(Suppl 3):S2-62.

6. Brain Trauma Foundation American Association of Neurological Surgeons Congress of Neurological Surgeons. Guidelines for the management of severe traumatic brain injury. 7 Neurotrauma 2007;24(Suppl 1):S1-106.

7. Wakai A, Roberts IG, Schierhout G. Mannitol for acute traumatic brain injury. Cochrane Database Syst Rev 2008;8: CD001049.

8. Sakowitz OW, Stover JF, Sarrafzadeh AS, et al. Effects of mannitol bolus administration on intracranial pressure, 
cerebral extracellular metabolites, and tissue oxygenation in severely head-injured patients. 7 Trauma Acute Care Surg 2007;62(2):292-8.

9. McGraw PC, Howard G. Effect of mannitol on increased intracranial pressure. Neurosurgery 1983;13(3):269-71.

10. Barbic D, Barbic S, Lang E, et al. Hypertonic saline in acute traumatic brain injury? A systematic review and meta-analysis. CFEM 2010;12(3):231.

11. Kamel H, Navi BB, Nakagawa K, et al. Hypertonic saline versus mannitol for the treatment of elevated intracranial pressure: a meta-analysis of randomized clinical trials*. Crit Care Med 2011;39(3):554-9.

12. Lazaridis C, Neyens R, Bodle J, et al. High-osmolarity saline in neurocritical care: systematic review and metaanalysis. Crit Care Med 2013;41(5):1353-60.

13. Rickard AC, Smith JE, Newell P, et al. Salt or sugar for your injured brain? A meta-analysis of randomised controlled trials of mannitol versus hypertonic sodium solutions to manage raised intracranial pressure in traumatic brain injury. Emerg Med 7 2014;31(8):679-83.

14. Mortazavi MM, Romeo AK, Deep A, et al. Hypertonic saline for treating raised intracranial pressure: literature review with meta-analysis. $\mathcal{f}$ Neurosurg 2012;116(1): 210-21.

15. Wade CE, Grady JJ, Kramer GC, et al. Individual patient cohort analysis of the efficacy of hypertonic saline/dextran in patients with traumatic brain injury and hypotension. 7 Trauma Acute Care Surg 1997;42(5 Suppl):S61-5.

16. Guyatt G, Rennie D, Meade M, et al. Users' guides to the medical literature: a manual for evidence-based clinical practice, 2nd ed. New York: McGraw-Hill; 2008.

17. Worster A. Home - BEEM - Best evidence in emergency medicine; 2016. Available at: https://beemcme.com/ (accessed 15 April 2016).
18. Milne K. SGEM\#150: hypertonic saline for traumatic brain injury; 2016. Available at: http://thesgem.com/2016/ 03/sgem 150-hypertonic-saline-for-traumatic-brain-injury/ (accessed 16 April 2016).

19. Wordpress. Jetpack by WordPress.com; 2016. Available at: https://wordpress.org/plugins/jetpack/ (accessed 15 April 2016).

20. Facebook. How is reach defined for each of my Page posts?; 2016. Available at: https://www.facebook.com/help/ 241332825914969 (accessed 15 April 2016).

21. Symplure LLC. Symplur - connecting the dots in healthcare social media; 2016. Available at: symplur.com (accessed 15 April 2016).

22. Altmetric. Altmetric; 2016. Available at: https://www. altmetric.com (accessed 15 April 2016).

23. Trueger NS, Thoma B, Hsu CH, et al. The altmetric score: a new measure for article-level dissemination and impact. Ann Emerg Med 2015;66(5):549-53.

24. Luckett-Gatopoulos S, Thoma B, Milne K, et al. SGEM Hot Off the Press: regional nerve blocks for hip and femoral neck fractures: a systematic review. CFEM 2016;18(4): 296-300.

25. Milne K. SGEM \#138: hip to be blocked - regional nerve blocks for hip and femoral neck fractures; 2015. Available at: http://thesgem.com/2015/11/sgem138-hip-to-be-blockedregional-nerve-blocks-for-hip-and-femoral-neck-fractures/ (accessed 15 April 2016).

26. Lulic I, Kovic I. Analysis of emergency physicians' Twitter accounts. Emerg Med 7 2013;30(5):371-6.

27. Chan TM, Rosenberg H, Lin M. Global Emergency Medicine Journal Club: social media responses to the January 2014 online Emergency Medicine Journal Club on subarachnoid hemorrhage. Ann Emerg Med 2014; 64(1):88-94. 